09,13

\title{
Структурные и оптические свойства одинарных и бинарных пленок серебра и золота
}

\author{
(C) Г.М. Янковский ${ }^{1,2}$, А.В. Комаров ${ }^{1,3}$, Р.С. Пузько ${ }^{1,3}$, А.В. Барышев ${ }^{1,3,4}$, К.Н. Афранасьев ${ }^{1,5,6}$, \\ И.А. Богинская ${ }^{1,5,6}$, И.В. Быков ${ }^{1,5,6}$, А.М. Мерзликин ${ }^{1,3,5}$, И.А. Родионов ${ }^{1,6}$, И.А. Рыжиков ${ }^{1,5,6}$
}

${ }^{1}$ Всероссийский научно-исследовательский институт автоматики им. Н.Л. Духова,

Москва, Россия

${ }^{2}$ Национальный исследовательский технологический университет „МИСиС“,

Москва, Россия

${ }^{3}$ Московский ффизико-технический институт (Государственный университет),

Долгопрудный, Россия

${ }^{4}$ Физико-технический институт им. А.Ф. Иофффе РАН,

Санкт-Петербург, Россия

${ }^{5}$ Институт теоретической и прикладной электродинамики РАН,

Москва, Россия

${ }^{6}$ Московский государственный технический университет им. Н.Э. Баумана,

Москва, Россия

E-mail: gyankovskii@mail.ru

(Поступила в Редакцию 4 марта 2016 г.

В окончательной редакции 4 мая 2016 г.)

\begin{abstract}
Проведено экспериментальное исследование структурных и оптических свойств тонких пленок серебра, золота и бинарных пленок на основе этих металлов. Для изучения свойств нанопленок исследованы спектры отражения при возбуждении поверхностных плазмонов в геометрии Кречманна и спектры эллипсометрических параметров. Спектры отражения анализировались с помощью теоретической модели для определения (эффективных) диэлектрических постоянных пленок. Вычисленные величины диэлектрических постоянных пленок отличаются от данных, полученных методом эллипсометрии. Обсуждаются особенности определения диэлектрических постоянных разными методами и характеристики изготовленных пленок, связанные с влиянием подложки.
\end{abstract}

Работа выполнена при поддержке Фонда перспективных исследований (договор № 7/004/2013-2018 от 23.12.2013).

\section{1. Введение}

Успехи в нанофотонике связаны с появлением искусственных материалов, обладающих новыми оптическими свойствами [1]. Представителями таких материалов являются металлические структуры, оптические спектры которых определяются возбуждением поверхностного плазмон-поляритона (поверхностным и локализованным плазмонным резонансом) [2] — возбуждением плотности свободных зарядов под действием световой волны. Вследствие этих резонансов плазмонные структуры привносят сильные затухающие ближние поля в современные исследования [3], например позволяют создать миниатюрные оптические системы [4,5], повышают чувствительность биосенсоров [6,7], а также позволяют создать лазеры на основе поверхностных плазмонполяритонов [8].

Особое внимание уделяется изучению оптических свойств плазмонных материалов [9], так как именно диэлектрическая постоянная является ключевым параметром для предсказания свойств плазмонных структур на основе благородных металлов, нитрида титана, нитрида циркония, проводящих оксидов и других пер- спективных материалов. Металлические пленки толщиной более $100 \mathrm{~nm}$ имеют воспроизводимые структурные параметры (толщину, размер кристаллитов и шероховатость $[10,11])$, их оптические свойства довольно хорошо изучены [12-14]. Исторически сложилось так, что наиболее известны и пользуются широкой популярностью справочные данные по оптическим константам металлов, приведенные в работах [15-17]. Однако результаты [15] получены на основе анализа коэффициентов отражения и пропускания поликристаллических пленок толщиной от 20 до $50 \mathrm{~nm}$. Известно, что такие пленки, как правило, имеют шероховатость порядка нескольких нанометров и структурно значительно отличаются от толстых пленок $[18,19]$.

Исследование оптических констант металлов попрежнему актуально, что подтверждается регулярным появлением новых публикаций [20-22]. Пленки плазмонных материалов, изготовленные на конкретном технологическом оборудовании при использовании различных подложек и режимов напыления, являются уникальными и требуют детальной характеризации.

Известно, что в оптическом диапазоне частот лучшими плазмонными свойствами обладают серебро и 
золото; из этой пары лучшим считается серебро [23-26]. Однако серебро имеет существенный недостаток: оно не достаточно стойко к агрессивным средам и характеризуется свойством хемосорбции различных веществ на его поверхности. В реальных условиях неизбежно происходит поверхностная модификация серебряных пленок, этот процесс может происходить как за короткое время, так и в течение нескольких дней [27]. Для поддержания качества поверхности пленок серебра их покрывают защитным слоем. Защитный слой может быть прозрачным (например, кварц [28] или оксид алюминия [29]) или непрозрачным (золото [30]). Для многослойных образцов на основе серебра по-прежнему важна характеризация структурных и оптических свойств, поскольку на границе раздела может происходить модификация физических свойств слоя серебра.

Отметим, что методы характеризации оптических свойств, основанные на анализе коэффициентов отражения/пропускания, чувствительны к морфологии поверхности образца $[15,31]$. Морфология поверхности в значительной степени влияет на уровень плазмонных потерь, т.е. на спектр поверхностного плазмонного резонанса (ППР). В свою очередь ППР можно использовать для анализа структурных особенностей плазмонных пленок, т. е. можно использовать принцип плазмонного микроскопа [32]. Данный метод обеспечивает непосредственное измерение эффективности трансформации энергии электромагнитных волн в энергию поверхностных плазмонов в зависимости от структуры поверхности образца. В этой работе реализация принципа плазмонного микроскопа основана на анализе изменения коэффициента отражения в геометрии Кречманна [33] при сканировании поверхности образца сфокусированным лазерным лучом.

В настоящей работе проведены исследования морфологии поверхности одинарных пленок серебра и золота, а также бинарных пленок на основе этих материалов с помощью атомно-силовой микроскопии (АСM). Определены (эффективные) диэлектрические постоянные данных пленок с помощью эллипсометрии и при измерении оптических спектров в режиме возбуждения ППР. Демонстрируется изменение диэлектрической постоянной пленки серебра во времени. Предложен критерий характеризации качества пленок, выращенных на различных подложках, и обсуждается подход для улучшения их структурных и оптических свойств.

\section{2. Образцы и эксперимент}

В работе были исследованы пленки серебра и золота, а также бинарные пленки на основе этих металлов, изготовленные на плоских стеклянных подложках толщиной $1 \mathrm{~mm}$ и площадью $4 \mathrm{~cm}^{2}$. На подложки напылялся либо слой хрома толщиной $h_{\mathrm{Cr}} \approx 3 \mathrm{~nm}$ для повышения адгезии, либо выравнивающий слой $\mathrm{SiO}_{2}$ толщиной $h_{\mathrm{SiO}_{2}} \approx 75 \mathrm{~nm}$. Толщина бинарных пленок была равна $d_{\mathrm{Ag}}+d_{\mathrm{Au}} \approx 45 \mathrm{~nm}$, при этом варьировались толщины пленок серебра $d_{\mathrm{Ag}}$ и золота $d_{\mathrm{Au}}$ в диапазоне $10-35 \mathrm{~nm}$, для данной серии также были изготовлены пленки серебра и золота толщиной $d \approx 45-55 \mathrm{~nm}$. Исследованные образцы были изготовлены методом электронно-лучевого распыления с помощью установки УРМ3.279.072. Напыление происходило при высоком вакууме (давление $2 \cdot 10^{-5}$ Torr), скорость напыления $10 \AA /$ s. Для напыления металлов и $\mathrm{SiO}_{2}$ использовались мишени с чистотой 99.99\%.

Для характеризации структурных свойств и определения толщины образца и шероховатости использовались атомно-силовой микроскоп Solver P47-PRO и оптический интерференционный топограф (Zygo NewView $7300)$, работающий на длине волны $475 \mathrm{~nm}$. Точность определения толщины напыленной пленки с помощью топографа составляла $\pm 0.5 \mathrm{~nm}$.

Измерение эллипсометрических параметров проводилось в диапазоне $350-1050 \mathrm{~nm}$ с помощью эллипсометрического комплекса САГ-1891 (Новосибирск), оснащенного программным обеспечением для определения диэлектрических постоянных ( $\varepsilon^{\prime}$ и $\left.\varepsilon^{\prime \prime}\right)$. Эксперименты проводились при двух углах падения излучения на поверхность образца: $\alpha=45$ и 70 $(\alpha-$ угол между лучом и нормалью к поверхности исследуемой пленки). Диаметр светового пятна на поверхности пленки составлял $\sim 5 \mathrm{~mm}$.

Наблюдение ППР в металлических пленках проводилось в геометрии Кречманна [33]. Возбуждение поверхностного плазмона осуществлялось р-поляризованным светом от лазерных источников на длинах волн 632.8 и $655 \mathrm{~nm}$. В отличие от экспериментов с помощью эллипсометра в этих экспериментах диаметр светового пятна на образце был $\sim 0.2-0.3 \mathrm{~mm}$. Для изменения угла падения света на образец использовалась вращающаяся платформа с шагом вращения $0.03^{\circ}$. В эксперименте измерялась зависимость коэффициента отражения от угла падения света на образец $\beta$ ( $\beta$ - угол внутри призмы относительно нормали к поверхности образца). Интенсивность отраженного света регистрировалась с помощью фотодетектора, сопряженного с интегрирующей сферой. Для анализа свойств изготовленного образца его различные области сканировались лазерным лучом. Последнее достигалось с помощью линейного транслятора, смещающего призму с образцом вдоль оси, перпендикулярной плоскости падения, без изменения угла $\beta$.

Экспериментальные спектры аппроксимировались численно с помощью оригинального алгоритма, использующего матрицы переноса [34]. Исследуемый образец в расчетах моделировался как один однородный изотропный слой, расположенный на поверхности призмы. При расчетах параметры призмы играют существенную роль, поэтому были проведены дополнительные измерения оптических свойств призмы. Показатель преломления призмы был восстановлен по спектру полного внутреннего отражения. В качестве подгоночных параметров 
были выбраны толщина слоя $d$, действительная $\varepsilon^{\prime}$ и мнимая $\varepsilon^{\prime \prime}$ части диэлектрической постоянной. Критерием подбора этих параметров являлось достижение минимума невязки между экспериментальной и теоретической кривыми спектров отражения. В качестве меры невязки использовалась мера $L_{2}$ в диапазоне углов $\beta$, задаваемых геометрией эксперимента:

$$
S\left(\varepsilon^{\prime}, \varepsilon^{\prime \prime}, d\right)=\int_{\beta_{\min }}^{\beta_{\max }}\left|R_{\exp }(\beta)-R_{\mathrm{th}}\left(\beta, \varepsilon^{\prime}, \varepsilon^{\prime \prime}, d\right)\right|^{2} d \beta,
$$

где $R_{\exp }(\beta)$ - экспериментальная зависимость коэффициента отражения от угла падения, а $R_{\mathrm{th}}\left(\beta, \varepsilon^{\prime}, \varepsilon^{\prime \prime}, d\right)-$ теоретическая зависимость коэффициента отражения, вычисленная с помощью матриц переноса, $\beta_{\min }$ и $\beta_{\max }-$ минимальный и максимальный углы падения луча из призмы на исследуемый слой. Для нахождения минимума невязки использовался метод градиентного спуска [35]. Для данного метода необходимо выбрать начальное приближение. С этой целью на сетке параметров производился поиск локальных минимумов невязки, которые использовались в качестве начальных значений. По толщине слоя металла сетка имела размер от 25.0 до $70.0 \mathrm{~nm}$ (с шагом $0.15 \mathrm{~nm}$ ), по действительной части диэлектрической проницаемости металла - от -25.0 до 0 (с шагом 0.125), по мнимой части - от 0 до 5.0 (с шагом 0.025). Такой размер сетки был выбран для того, чтобы покрыть предположительные параметры. Мелкость разбиения сетки варьировалась, чтобы убедиться, что она достаточна для обнаружения всех локальных минимумов.

В спектре отражения, характерном для поверхностного плазмона на бинарной пленке, можно выделить три существенные особенности: ширина плазмонного резонанса, его положение и минимальное значение коэффициента отражения. Данные параметры очень точно характеризуют кривую отражения. Поэтому подгонка трех параметров (толщины, действительной и мнимой частей эффективной диэлектрической проницаемости) позволяет точно аппроксимировать экспериментальные спектры отражения. Использование шести подгоночных параметров (по три для каждого из слоев металла) является превышением точности, при котором малость невязки конкурирует с эффектами неровности пленки и неточности измерений. Поэтому подгонка параметров каждого из слоев бинарной системы является нецелесообразной.

\section{3. Результаты и обсуждение}

Полученные пленки имели островковую структуру с размерами кристаллитов $D \approx 30-90 \mathrm{~nm}$ (рис. 1). Среднеквадратичная шероховатость пленок (root mean square, RMS) измерялась с помощью АCM. Следует отметить, что шероховатость пленок, нанесенных на адгезионный слой хрома $\mathrm{RMS}(\mathrm{Ag} / \mathrm{Cr})$, равная $\sim 5 \mathrm{~nm}$
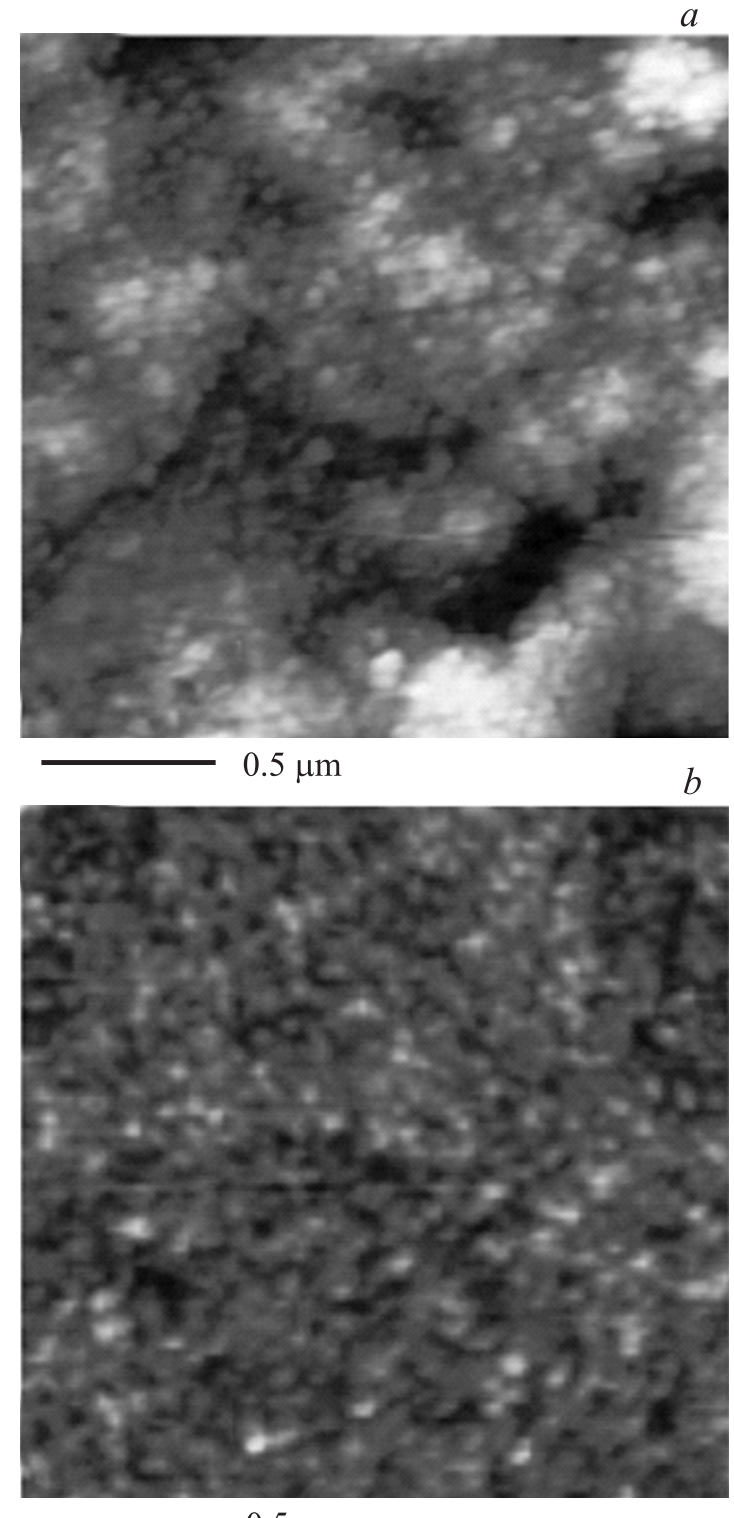

$0.5 \mu \mathrm{m}$

Рис. 1. АСМ-изображения поверхности серебра толщиной $45 \mathrm{~nm}$ на слое хрома $\left(h_{\mathrm{Cr}}=3 \mathrm{~nm}\right)$ (a) и слое $\mathrm{SiO}_{2}$ $\left(h_{\mathrm{SiO}_{2}}=75 \mathrm{~nm}\right)(b)$.

(рис. 1,a), всегда больше RMS пленок, нанесенных на выравнивающий слой $\mathrm{SiO}_{2}$. Для серебряной пленки толщиной $45 \mathrm{~nm}$ на выравнивающем слое $\mathrm{SiO}_{2}$, представленной на рис. $1, b, \mathrm{RMS}\left(\mathrm{Ag} / \mathrm{SiO}_{2}\right)=1.1 \mathrm{~nm}$, а размер кристаллита $D=53 \pm 11 \mathrm{~nm}$. Для пленки на адгезионном слое хрома $\mathrm{RMS}(\mathrm{Ag} / \mathrm{Cr})=5.26 \mathrm{~nm}$, размер кристаллита $D=56 \pm 8 \mathrm{~nm}$. Для бинарных пленок на слое $\mathrm{SiO}_{2}$ характерная величина $\mathrm{RMS}\left(\mathrm{Au} / \mathrm{Ag} / \mathrm{SiO}_{2}\right)$ была $1 \mathrm{~nm}$; в частности, для бинарной пленки с суммарной толщиной $45 \mathrm{~nm} \quad(\mathrm{Au}(20 \mathrm{~nm}) / \mathrm{Ag}(25 \mathrm{~nm}))$ $\mathrm{RMS}\left(\mathrm{Au} / \mathrm{Ag} / \mathrm{SiO}_{2}\right)=1.1 \mathrm{~nm}$, а размер кристаллита равен $D=56 \pm 5 \mathrm{~nm}$.

На рис. 2 представлены дисперсии диэлектрических постоянных $\left(\begin{array}{lll}\varepsilon^{\prime} & \text { и } & \varepsilon^{\prime \prime}\end{array}\right)$ для пленок серебра толщиной 


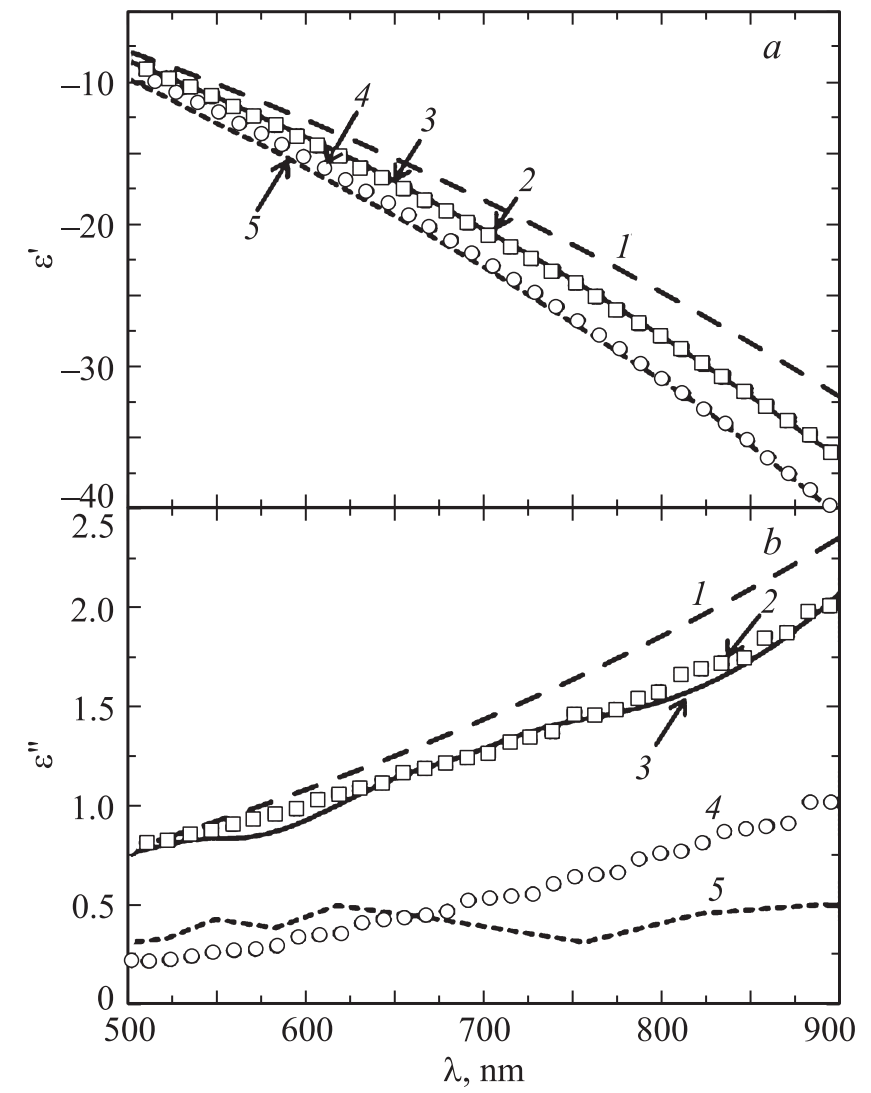

Рис. 2. Дисперсия диэлектрической постоянной пленок $\mathrm{Ag}$ толщиной $25.5 \mathrm{~nm}$ (точки 2) и $45 \mathrm{~nm}$ (точки 4). $a$ - действительная, $b-$ мнимая часть. Угол падения света на образец $\alpha=70^{\circ}$. Кривые соответствуют литературным данным для пленок Ag: $1-$ [17], $3-[16], 5-[15]$.

25.5 и 45 nm, напыленных на выравнивающий слой $\mathrm{SiO}_{2}$, полученные с помощью эллипсометра при наклонном падении света $\left(\alpha=70^{\circ}\right)$ относительно нормали к поверхности пленки. На рисунке также представлены литературные данные из работ [15-17]. Из рисунка видно, что экспериментальные данные для пленки толщиной $25.5 \mathrm{~nm}$ совпадают с данными работы [16], а для пленки толщиной $45 \mathrm{~nm}$ - с данными работы [15].

Аналогичные измерения проводились для пленки $\mathrm{Au}$ толщиной $36 \mathrm{~nm}$ (рис. 3). Наши исследования показали, что величины $\varepsilon^{\prime}$ и $\varepsilon^{\prime \prime}$ для данного образца зависели от угла падения света (см. вставки к рис. 3). Наиболее вероятно, что данный результат (и различия на рис. 2) является проявлением островковой структуры пленки. Действительно, экспериментальные зависимости, полученные при $\alpha=70^{\circ}$, находились в лучшем соответствии с литературными данными [15]. Величины комплексной составляющей диэлектрической постоянной в диапазоне длин волн до $655 \mathrm{~nm}$ меньше, чем табличные данные из работы [15]. При увеличении длины волны $\varepsilon^{\prime \prime}$ начинает превышать данные как [15], так и [17]. Следует упомянуть, что данные работы [15] также получены для тонких пленок.

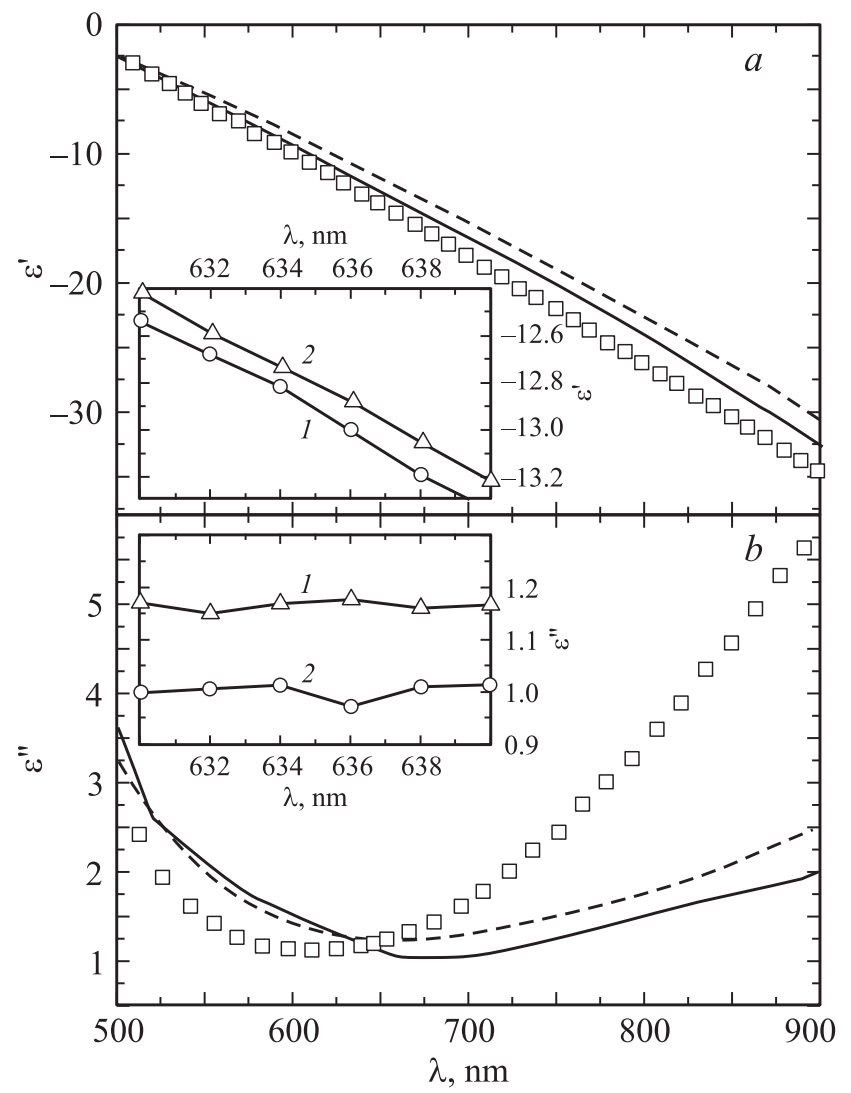

Рис. 3. Дисперсия диэлектрической постоянной пленки $\mathrm{Au}$ толщиной $36 \mathrm{~nm}$ (точки) для $\alpha=70^{\circ} . a-$ действительная, $b$ - мнимая часть. На вставках - кривые 1 и 2 отвечают $\alpha=45$ и $70^{\circ}$ соответственно. Сплошная кривая соответствует литературным данным для Аu из работы [15], штриховая данным из работы [17].

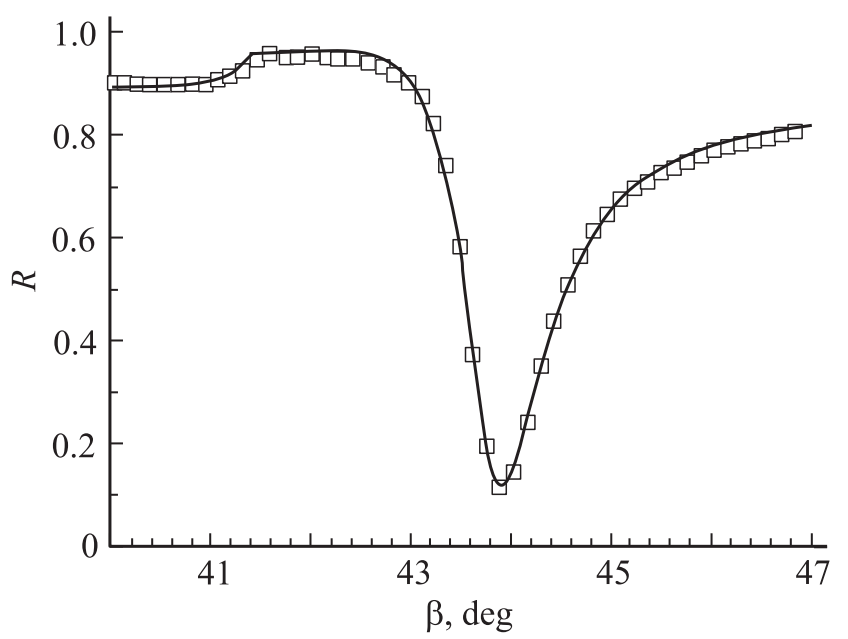

Рис. 4. Пример аналитической обработки экспериментальной зависимости для пленки $\mathrm{Au}(d \approx 45 \mathrm{~nm})$, выращенной на выравнивающем слое $\mathrm{SiO}_{2}\left(h_{\mathrm{SiO}_{2}}=75 \mathrm{~nm}\right)$. Линией показана расчетная зависимость, дающая наименьшее расхождение с экспериментальной зависимостью (точки). Рабочая длина волны лазера $\lambda=632.8 \mathrm{~nm}$. 


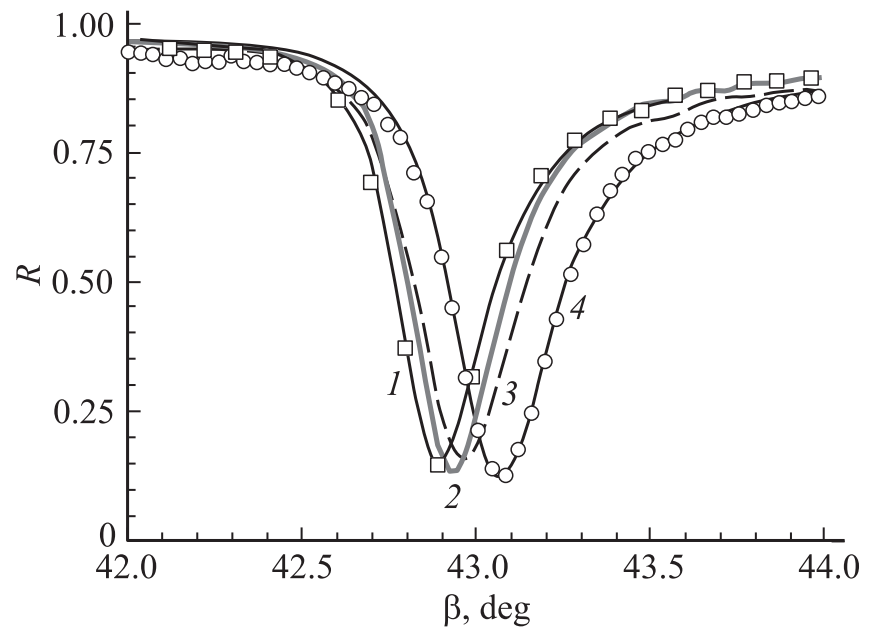

Рис. 5. Зависимости коэффициента отражения от угла падения для пленки $\mathrm{Ag}(d \approx 52 \mathrm{~nm})$. Кривая 1 измерена через $6 \mathrm{~h}$ после изготовления, 2 - через неделю, 3 - через месяц, 4 - через четыре месяца после изготовления. Для кривых 1 и 4 тонкими линиями показаны теоретические зависимости. Рабочая длина волны лазера $\lambda=655 \mathrm{~nm}$.

На рис. 4 приведен пример обработки экспериментальных данных по наблюдению ППР. Теоретический анализ зависимости показал, что пленка характеризуется следующими параметрами: $d=46.8 \mathrm{~nm}$, $\varepsilon^{\prime}=-11.1$ и $\varepsilon^{\prime \prime}=0.66$. Отметим, что оценка толщины данной пленки $\mathrm{Au}$ с помощью топографа дала величину $d=45.0 \pm 0.5 \mathrm{~nm}$, а $\varepsilon^{\prime}=-12.25$ и $\varepsilon^{\prime \prime}=0.50$ для длины волны $\lambda=632.8 \mathrm{~nm}$ установлены с помощью эллипсометра. Из полученных значений видно, что эллипсометрия и наш аналитический подход дали близкие величины, а при определении толщины пленки обнаружено совпадение с точностью $1 \mathrm{~nm}$. Однако нужно подчеркнуть, что различия в величинах $\varepsilon^{\prime}$ и $\varepsilon^{\prime \prime}$, по-видимому, являются следствием перечисленных далее факторов. Во-первых, рассеяние поверхностного плазмона на шероховатостях поверхности пленки в экспериментах по наблюдению ППР могло повлиять на ширину и интенсивность детектируемой полосы поглощения. Во-вторых, в эллипсометрических экспериментах засвечивалась значительная область на поверхности пленки (размер пятна $3 \times 9 \mathrm{~mm}$ ), тогда как в экспериментах по наблюдению ППР диаметр пятна засветки был на порядок меньше. Следовательно, полученные величины можно рассматривать как оценку усредненной и локальной величины диэлектрических постоянных напыленного золота.

Для выбранной пленки $\mathrm{Ag}(d \approx 52 \mathrm{~nm}$ - величина определенная топографом), изготовленной на стеклянной подложке, наблюдение ППР проводилось как непосредственно после ее изготовления, так и через продолжительные промежутки времени. Пленка хранилась в закрытой пластиковой коробке на воздухе при комнатной температуре. Результаты этих экспериментов представлены на рис. 5. Угловые зависимо- сти коэффициента отражения света с $\lambda=655 \mathrm{~nm}$ были получены через $6 \mathrm{~h}$ после напыления (кривая 1), через неделю (кривая 2), через месяц (кривая 3) и через четыре месяца (кривая 4). Видно, что минимум ППР сдвигается в сторону больших углов с течением времени. По результатам аналитической обработки такое смещение соответствует следующим величинам: кривая $1-d=55.8 \mathrm{~nm}, \varepsilon^{\prime}=-17.66$ и $\varepsilon^{\prime \prime}=1.07$; кривая $2-d=56.1 \mathrm{~nm}, \varepsilon^{\prime}=-17.17$ и $\varepsilon^{\prime \prime}=0.98$; кривая $3-d=56.8 \mathrm{~nm}, \varepsilon^{\prime}=-16.96$ и $\varepsilon^{\prime \prime}=1.09$; кривая $4-d=57.8 \mathrm{~nm}, \varepsilon^{\prime}=-15.99$ и $\varepsilon^{\prime \prime}=1.01$. Из этих данных видно, что со временем абсолютная величина $\varepsilon^{\prime}$ уменьшилась на 9.4\%. Как показано далее, исследованные пленки имеют неоднородность свойств в различных областях. Поэтому в экспериментах, представленных на рис. 5, область засветки пленки была фиксированной.

Для анализа свойств изготовленных металлических пленок различные области образца сканировались лазерным лучом, как описано в разделе 2. В этом эксперименте использовалась высокая чувствительность ППР как к малейшим флуктуациям толщины пленки, так и к флуктуациям показателя преломления материала на поверхности пленки. На рис. 6, a показаны угловые зависимости коэффициента отражения для двух разных областей образца - одинарной пленки $\mathrm{Ag}$ $(d \approx 52 \mathrm{~nm})$, напыленной на стеклянную подложку. Ступенчатая кривая на рис. $6, a$, соответствующая кривой 3 на рис. $6, b$, иллюстрирует коэффициент отражения при сканировании поверхности пленки с шагом $0.5 \mathrm{~mm}$ для угла $\beta$ в окрестности минимума полосы возбуждения ППР. В нашем случае эксперименты проводились в обычных комнатных условиях, и, конечно, загрязнение поверхности пленки было возможным. Однако наиболее вероятно, что обнаруженные смещения полосы ППР были связаны с флуктуациями толщины пленки. Свидетельством этого являются результаты аналитической обработки коэффициента отражения для двух разных областей образца (два контура полосы ППР): $d=56.1 \mathrm{~nm}$, $\varepsilon^{\prime}=17.17$ и $\varepsilon^{\prime \prime}=0.98$ и $d=56.9 \mathrm{~nm}, \varepsilon^{\prime}=-17.20$ и $\varepsilon^{\prime \prime}=0.99$. В данном случае видно, что изменение коэффициента отражения в точке минимума спектра на величину 0.02 соответствует изменению толщины серебряной пленки на $0.8 \mathrm{~nm}$. Таким образом, ступенчатая кривая на рис. $6, a$ иллюстрирует неоднородность пленки по толщине. Наибольшее изменение толщины пленки в диапазоне сканирования может достигать нескольких нанометров. Результаты сканирования лучом для трех образцов, выращенных на по-разному приготовленных подложках, показаны на рис. $6, b$. Видно, что относительное отклонение коэффициента отражения $\langle R\rangle=\Delta R / R_{a}$ для образца, выращенного на слое $\mathrm{SiO}_{2}$, было существенно меньше; $\Delta R=R_{\max }-R_{\min }, R_{\max }$ и $R_{\min }-$ максимальное и минимальное значения коэффициента отражения, измеренные при сканировании различных областей пленок, а $R_{a}$ - среднее арифметическое всех величин $R$ при сканировании. Такая оценка структурной однородности поверхности пленок $\mathrm{Ag}$ дала следующие 

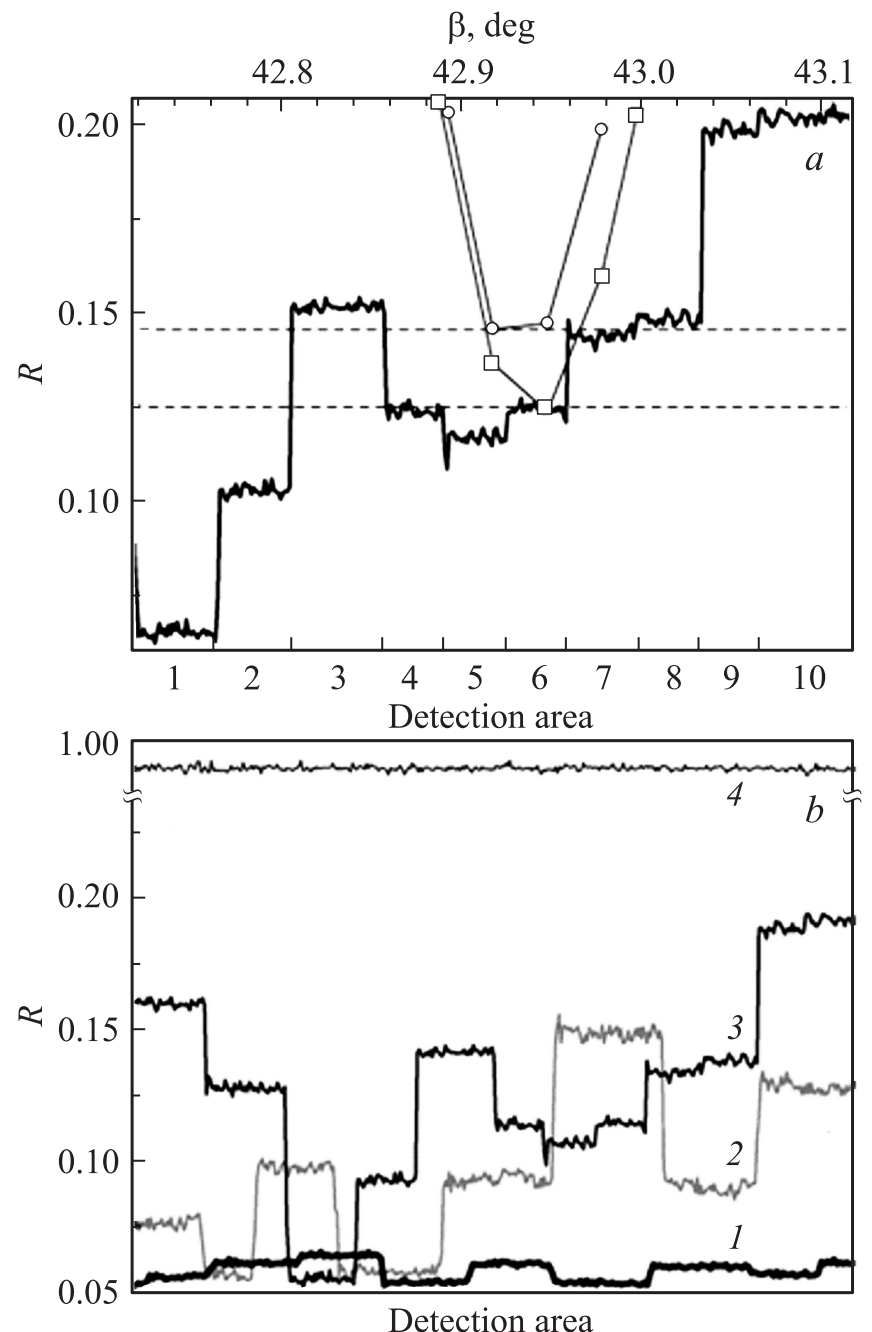

Рис. 6. $a)$ Угловые зависимости коэффициента отражения от разных областей пленки $\mathrm{Ag}(d \approx 52 \mathrm{~nm})$. Рабочая длина волны лазера $\lambda=655 \mathrm{~nm}$. Ступенчатая кривая иллюстрирует коэффициент отражения при сканировании поверхности пленки с шагом $0.5 \mathrm{~mm}$ для угла $\beta$ в окрестности минимума полосы возбуждения ППР (также приведена на панели $b$, кривая 3 ). $b$ ) Зависимости коэффициента отражения при сканировании поверхности образца на примере различных пленок $\mathrm{Ag}: 1-\operatorname{Ag}(d \approx 55 \mathrm{~nm}) /$ выравнивающий слой $\mathrm{SiO}_{2} \quad\left(h_{\mathrm{SiO}_{2}} \approx 75 \mathrm{~nm}\right) /$ подложка, $2-\mathrm{Ag}(d \approx 45 \mathrm{~nm}) /$ $\operatorname{Cr}\left(h_{\mathrm{Cr}} \approx 3 \mathrm{~nm}\right) /$ подложка, $3-\operatorname{Ag}(d \approx 52 \mathrm{~nm}) /$ подложка. Кривая 4 - коэффициент отражения при сканировании поверхности стеклянной подложки и угле $\beta$ в окрестности минимума полосы возбуждения ППР.

величины: $\langle R\rangle=0.78$ для серебра, напыленного на стеклянную подложку, $\langle R\rangle=0.61$ для серебра, напыленного на адгезионный слой хрома, и $\langle R\rangle=0.11$ для серебра, напыленного на выравнивающий слой $\mathrm{SiO}_{2}$. Таким образом, эксперименты показали, что напыление на стеклянную подложку выравнивающего слоя $\mathrm{SiO}_{2}$ приводило к росту более однородной пленки Ag.

Развитием экспериментов с пленками серебра и золота были исследования свойств бинарных пленок на основе этих металлов. Целью данных экспериментов было изготовление бинарных пленок $\mathrm{Ag} / \mathrm{Au}$ (ceребро, покрытое золотом) с фиксированной толщиной $\left(d_{\mathrm{Ag}}+d_{\mathrm{Au}} \approx 45 \mathrm{~nm}\right)$, а также определение эффективных диэлектрических постоянных пленок в серии $\mathrm{Ag} \rightarrow \mathrm{Ag} / \mathrm{Au} \rightarrow \mathrm{Au}$. По замыслу, толщины пленок в образцах $\mathrm{Ag} / \mathrm{Au}$ были следующими: образец № $1-\operatorname{Ag}(45 \mathrm{~nm}) / \mathrm{Au}(0 \mathrm{~nm})$, образец № $2-$ $\operatorname{Ag}(35 \mathrm{~nm}) / \mathrm{Au}(10 \mathrm{~nm})$, образец № $3-\operatorname{Ag}(30 \mathrm{Hм}) /$ $\mathrm{Au}(15 \mathrm{Hм})$, образец № $4-\operatorname{Ag}(25 \mathrm{~nm}) / \mathrm{Au}(20 \mathrm{~nm})$, образец № $5-\operatorname{Ag}(20 \mathrm{~nm}) / \mathrm{Au}(25 \mathrm{~nm})$, образец № $6-\operatorname{Ag}(15 \mathrm{~nm}) / \mathrm{Au}(30 \mathrm{~nm})$, образец № 7 $\mathrm{Ag}(0 \mathrm{~nm}) / \mathrm{Au}(45 \mathrm{~nm})$. Вся серия была выращена на стеклянных подложках с выравнивающим слоем $\mathrm{SiO}_{2}$. $\mathrm{Pe}$ зультаты экспериментов и теоретического анализа для пленок серии представлены в таблице для $\lambda=632.8 \mathrm{~nm}$. Дисперсионные зависимости диэлектрических постоянных пленок серии представлены на рис. 7.

Из данных, представленных в таблице, видно, что в ряде случаев эллипсометрия и аналитическая модель позволяют определить толщину пленки с точностью до 1-2 nm. Важно отметить, что величины $\varepsilon^{\prime}$ и $\varepsilon^{\prime \prime}$, полученные в результате обработки угловых зависимо-

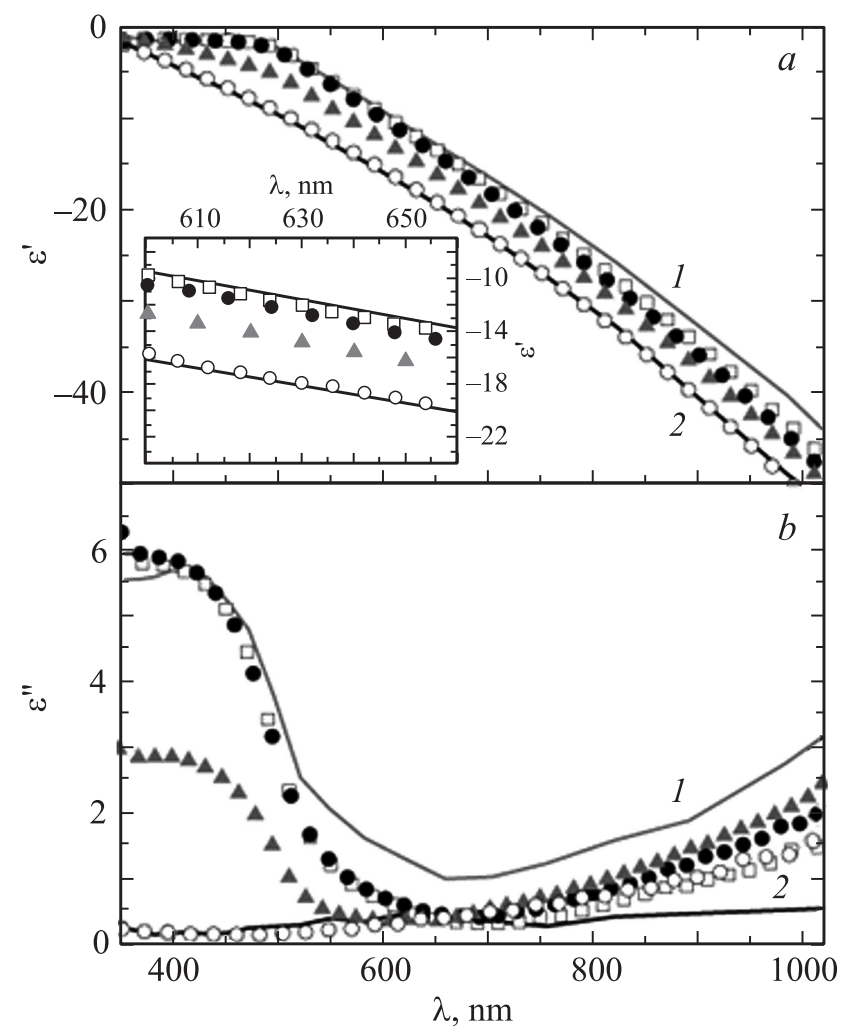

Рис. 7. а) Зависимость действительной части диэлектрической постоянной от длины волны для двухслойных структур с суммарной толщиной $\sim 45 \mathrm{~nm}$. $b$ ) Зависимость мнимой части диэлектрической постоянной. Светлые кружки - образец № 1 , треугольники - образец № 2, темные кружки - образец № 6, квадраты - образец № 7. Кривые - табличные данные [15] для пленок $\mathrm{Au}(1)$ и $\mathrm{Ag}(2)$. 
Эффективные диэлектрические постоянные бинарных пленок $\mathrm{Ag} / \mathrm{Au}$ на выравнивающем слое $\mathrm{SiO}_{2}$ (данные представлены для $\lambda=632.8 \mathrm{~nm})$

\begin{tabular}{|c|c|c|c|c|c|c|}
\hline \multirow{3}{*}{$\begin{array}{l}\text { Номер } \\
\text { образца }\end{array}$} & \multirow{3}{*}{$\begin{array}{c}\text { Толщина } \\
\text { измеренная } \\
\text { топографом, } \\
\text { nm }\end{array}$} & \multirow{3}{*}{$\begin{array}{c}\text { Вычисленная } \\
\text { толщина, } \\
\text { nm }\end{array}$} & \multicolumn{4}{|c|}{ Эффективные диэлектрические постоянные } \\
\hline & & & \multicolumn{2}{|c|}{$\begin{array}{c}\text { На основе результатов } \\
\text { эллипсометрии }\end{array}$} & \multicolumn{2}{|c|}{$\begin{array}{c}\text { На основе результатов } \\
\text { наблюдения ППР }\end{array}$} \\
\hline & & & $\varepsilon^{\prime}$ & $\varepsilon^{\prime \prime}$ & $\varepsilon^{\prime}$ & $\varepsilon^{\prime \prime}$ \\
\hline 1 & $43.0 \pm 0.5$ & 42.6 & -18.05 & 0.41 & -16.63 & 0.53 \\
\hline 2 & $39.3 \pm 0.5$ & 42.4 & -15.01 & 0.42 & -14.21 & 0.94 \\
\hline 3 & $40.5 \pm 0.5$ & 44.9 & -14.62 & 0.63 & -13.04 & 0.97 \\
\hline 4 & $36.7 \pm 0.5$ & 38.9 & -13.68 & 0.37 & -12.91 & 1.22 \\
\hline 5 & $37.0 \pm 0.5$ & 40.1 & -13.72 & 0.33 & -12.19 & 1.04 \\
\hline 6 & $37.3 \pm 0.5$ & 42.8 & -12.80 & 0.57 & -11.94 & 1.07 \\
\hline 7 & $45.0 \pm 0.5$ & 46.8 & -12.25 & 0.50 & -11.10 & 0.66 \\
\hline
\end{tabular}

стей коэффициента отражения, отличались от величин, полученных с помощью эллипсометра. Таким образом, меньшая абсолютная величина $\varepsilon^{\prime}$ и бо́льшая $\varepsilon^{\prime \prime}$ отражают ухудшение „плазмонных свойств“ изготовленных пленок, т.е. диссипацию плазмон-поляритона из-за рассеяния на шероховатостях поверхности пленок. Из рис. 7 видно, что дисперсионные зависимости $\varepsilon^{\prime}$ и $\varepsilon^{\prime \prime}$ для бинарных пленок находятся внутри диапазона величин для одинарных пленок $\mathrm{Ag}$ и $\mathrm{Au}$, которые в свою очередь совпадают или близки к результатам работы [15]. Более наглядно различия между данными по $\varepsilon^{\prime}$ для бинарных пленок с различной толщиной слоев иллюстрируются на вставке к рис. $7, a$.

\section{4. Заключение}

Различными методами исследованы структурные и оптические свойства тонких пленок серебра и золота, a также бинарных пленок на основе этих металлов. В работе определены (эффективные) диэлектрические постоянные пленок. Демонстрируется, что величины диэлектрических постоянных тонких пленок, полученные с использованием эллипсометра, имеют незначительную зависимость от геометрии эксперимента. Величины диэлектрических постоянных, установленные с помощью теоретического анализа угловых зависимостей коэффициента отражения в режиме возбуждения поверхностного плазмонного резонанса (геометрия Кречманна), незначительно отличались от данных эллипсометрии и литературных данных. Обнаруженное отличие, повидимому, является следствием дополнительного рассеяния плазмон-поляритона как на границе раздела пленка/воздух, так и на границе $\mathrm{Ag} / \mathrm{Au}$.

Показано, как оптические свойства тонких пленок серебра меняются с течением времени и как структурные свойства пленок зависят от подготовки подложки. Исследования свойств поверхности пленок с помощью АCM находятся в хорошем согласии с выводами, сделанными на основе оптических экспериментов. В качестве наиболее перспективных плазмонных пленок предложены бинарные пленки со структурой стеклянная подложка/выравнивающий слой $\mathrm{SiO}_{2} / \mathrm{Ag} / \mathrm{Au}$. Показано, что с помощью выбора относительных толщин слоев серебра и золота можно контролировать эффективную диэлектрическую проницаемость бинарной пленки в диапазоне величин, характерных для серебра и золота. Изучение временно́й устойчивости оптических свойств бинарных пленок является предметом будущего исследования.

\section{Список литературы}

[1] Active plasmonics and tunable plasmonic metamaterials / Eds A.V. Zayats, S. Mayer. Wiley and Science Wise Publ. (2013). $336 \mathrm{p}$.

[2] H. Raether. Surface plasmons on smooth and rough surfaces and on grating. Springer-Verlag, Berlin-Heidelberg (1988). $135 \mathrm{p}$.

[3] S.A. Maier. Plasmonics: fundamentals and applications. Springer, N.Y. (2007). 224 p.

[4] S.I. Bozhevolnyi, V.S. Volkov, E. Devaux, J.-Y. Laluet, T.W. Ebbesen. Nature 440, 508 (2006).

[5] T.W. Ebbesen, C. Genet, S.I. Bozhevolnyi. Phys. Today 61, 44 (2008).

[6] R. Slavik, J. Homola, H. Vaisocherova. Meas. Sci. Technol. 17, 932 (2006).

[7] A.V. Baryshev, A.M. Merzlikin, M. Inoue. J. Phys. D 46, 125107 (2013).

[8] P. Berini, I. De Leon. Nature Photon. 6, 16 (2012).

[9] Reviews in plasmonics 2010 / Ed. C.D. Geddes. SpringerVerlag, N.Y. (2012). 334 p.

[10] K.M. McPeak, S.V. Jayanti, S. Kress, S. Meyer, S. Iotti, A. Rossinelli, D. Norris. ACS Photon. 2, 326 (2015).

[11] V.A. Fedotov, T. Uchino, J.Y. Ou. Opt. Express 20, 9545 (2012).

[12] R.M.A. Azzam, N.M. Bashara. Ellipsometry and polarized light. North-Holland Publ. Co., Amsterdam (1977). 529 p.

[13] M. Losurdo. Thin Solid Films 519, 2575 (2011).

[14] N.P. Blanchard, C. Smith, D.S. Martin, D.J. Hayton, T.E. Jankins, P. Weightman. Phys. Status Solidi C 0, 8, 2931 (2003).

[15] P.B. Johnson, R.W. Christy. Phys. Rev. B 6, 4370 (1972). 
[16] Handbook of optical constants of solids / Ed. E.D. Palik. Academic, San Diego (1985). V. 1. 804 p.

[17] A.D. Rakic, A.B. Djurisic, J.M. Elazar, M.L. Majewski. Appl. Opt. 37, 5271 (1998).

[18] D.E. Aspnes, E. Kinsbron, D.D. Bacon. Phys. Rev. B 21, 3290 (1980).

[19] V.P. Drachev, U.K. Chettiar, A.V. Kildishev, H.-K. Yuan, W. Cai, V.M. Shalaev. Opt. Express 16, 1186 (2008).

[20] S. Babar, J.H. Weaver. Appl. Opt. 54, 477 (2015).

[21] A.W. Signor, J.H. Weaver. Phys. Rev. B 84, 165441 (2011).

[22] O. Kvitek, J. Siegel, V. Hnatowicz, V. Svorcik. J. Nanomater. 2013, 743684 (2013).

[23] P.R. West, S. Ishii, G.V. Naik, N.K. Emani, V.M. Shalaev, A. Boltasseva. Laser Photon. Rev. 4, 795 (2010).

[24] V.J. Keast, B. Zwan, S. Supansomboon, M.B. Cortie, P.O.A. Persson. J. Alloys and Compd. 577, 581 (2013).

[25] M.G. Blaber, M.D. Arnold, M.J. Ford. J. Phys.: Condens. Matter 22, 095501 (2010).

[26] G.V. Naik, J. Kim, A. Boltasseva. Opt. Mater. Express 1, 1090 (2011).

[27] А.А. Соловьев, Н.С. Сочугов, К.В. Оскомов. Изв. вузов. Физика. 9 (Приложение), 394 (2007).

[28] J. Sancho-Parramon, M. Pavlovic, H. Zorc, P. Dubcek, A. Turkovic, S. Bernstorff, G. Jakopic, A. Haase, M. Loncaric. Vacuum 84, 188 (2010).

[29] G.B. Smith, A.I. Maaroof. Opt. Commun. 242, 383 (2004).

[30] T. Lopez-Rios, G. Vuye. Surf. Sci. 81, 529 (1979).

[31] Д.И. Биленко, А.А. Сагайдачный, В.В. Галушка, В.П. Полянская. ЖТФ 80, 10, 89 (2010).

[32] С.И. Валянский. Сорос. образоват. журн. 8, 76 (1999).

[33] E. Kretschmann. Z. Phys. 241, 4, 313 (1971).

[34] М. Борн, Э. Вольф. Основы оптики. Наука, М. (1973). $720 \mathrm{c}$.

[35] И.Б. Петров, А.И. Лобанов. Лекции по вычислительной математике. Интернет-Университет информационных технологий, БИНОМ, Лаборатория знаний, М. (2006). 523 с. 